Original Research Paper

\title{
Local Large Deviations: A McMillian Theorem for Typed Random Graph Processes
}

\author{
Kwabena Doku-Amponsah \\ Department of Statistics, School of Physical and Mathematical Sciences, CBAS, University of Ghana, Ghana
}

Article history

Received: 08-07-2017

Revised: $29-10-2017$

Accepted: 24-11-2017

Email: kdoku-amponsah@ug.edu.gh

\begin{abstract}
For a finite typed graph on $n$ nodes and with type law $\mu$ on finite alphabet $\Gamma$, we define the spectral potential $\rho_{\lambda}(., \mu)$, of the graph. From the $\rho_{\lambda}(., \mu)$ we define the Kullback action or the divergence function, $\mathrm{H}_{\lambda}(\pi \|$ $v$ ), with respect to an empirical link measure, $\pi$, as the Legendre dual. For the finite typed random graph conditioned to have an empirical link measure $\pi$ and empirical type measure $\mu$, we prove a Local large Deviation Principle (LLDP), with rate function $\mathrm{H}_{\lambda}(\pi \| v)$ and speed $n$. From this LLDP, we obtain a full conditional large deviation principle and a weak variant of the classical McMillian Theorem for the typed random graph models. Given the typical empirical link measure, $\lambda_{\mu \otimes \mu}$, we show that, the

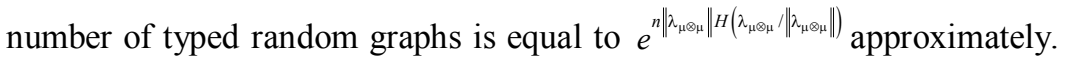
Note that we do not require any topological restrictions on the space of finite graphs for these LLDPs.
\end{abstract}

Keywords: Local Large Deviation Principle, Variational Principle, Empirical Link Measure, Spectral Potential Theory, Relative Entropy, Typed Random Process

\section{Introduction}

We consider random graph models, where nodes are assigned types independently according to some type on a finite alphabet and between any two given nodes, a link is present with a probability type on a finite alphabet and between any two given nodes, a link is present with a probability that depends on the type of the nodes. This random graph model was first proposed and studied extensively by Penman (1998) as a generalization of the Erdos-Renyi graphs. Indeed, the random graph model which is known to model fairly well network structured data, has the Erdos-Renyi graph as a special case. See, Cannings and Penman (2003) for an exposition on this random graphs and their applications.

Now, some Large Deviation Principles (LDPs) and Coding Theorems exists for networked data structures modelled as the Typed Random Graph (TRG) models. See, O'Connell (1998), Biggins and Penman (2009), Doku-Amponsah and Mörters (2010), Bordenave and Caputo (2005), Doku-Amponsah (2012, 2017a) and the reference therein. O'Connell (1998) proved Large Deviation Principle (LDP) for the relative size of the largest connected component in the random graph with small edge probability. Biggins and Penman (2009) have found LDPs for the number of edges of TRG models, where the link probabilities are independent of the number of nodes, using the Garten-Ellis Theorem, see Dembo and Zeitouni (1998). Doku-Amponsah and Mörters (2010) proved LDPs for the empirical measures of the TRG where the link probabilities are dependent on the number of nodes of the graph. In Bordenave and Caputo (2005), LDP for the empirical neighborhood distribution in sparse random graphs was proved using a technique that relies on the typical behavior within the framework of the local weak convergence of finite graph sequences. Asymptotic Equipartition Properties including the Lossy version have been found in Doku-Amponsah $(2012 ; 2017 \mathrm{a})$, by the techniques of exponential change of measure and random allocation, respectively.

We present in this article a conditional LLDP for the TRG models given the empirical type measure of the graph. Refer to Bakhtin (2015), Doku-Amponsah (2017) for similar results for the empirical measure of iid random variables and the empirical offspring measure of multitype Galton-Watson processes, respectively. This article shares similar features as Doku-Amponsah (2017c), but defers 
from all the LDPs discussed above. i.e., O'Connell (1998) Biggins and Penman (2009), Doku-Amponsah and Mörters (2010) Bordenave and Caputo (2005), Doku-Amponsah (2012; 2017b). The main technique used to prove our main result is rooted in spectral potential theory. See, DokuAmponsah (2017b) and the reference therein for similar idea for the LLDP for the multi-type Galton-Watson processes. To be precise about this technique, we define the spectral potential of the TRG and use it to calculate an extended version of the relative entropy and show that this relative entropy which has all the properties of the classical relative entropy, see Dembo and Zeitouni (1998), is the Legendre dual of our spectral potential of the TRG. From the LLDP for the TRG we deduce the weak variant of the classical McMillian Theorem and the full LDP for the TRG conditioned on the empirical link measure and under the conditional law of the TRG given the type law.

\section{Coloured Random Process}

Let $p_{n}: \Gamma \times \Gamma \rightarrow[0,1]$ be a symmetric function and $\mu$ on $\Gamma$ be a probability measure. We can define the typed random graph $Y$ with $[n]=\{1,2,3, \ldots, n\}$ nodes as follows:

- Allocate to each node $v \in[n]$ type $Y(v)$ independently according to the type law $\mu$

- For any pair of types $Y(v), Y(v)$, we connect two vertices $u, v \in[n]$, independently of everything else, with link probability $p_{n}(Y(u), Y(u))$

We always look at $Y=((Y(v): v \in[n]), E)$ with respect to the combine law of the graph model and type and interpret $Y$ as typed random graph model.

Denote by $\mathcal{G}([n], \Gamma)$ the set of all coloured graphs with colour set $\Gamma$ and $n$ vertices. We shall only study $Y$ with link probabilities satisfying:

$a_{n}^{-1} p_{n}(a, b) \rightarrow \lambda(a, b), \forall a, b \in \Gamma$,

where $\lambda: \Gamma \times \Gamma \rightarrow[0, \infty]$ is a nonzero function.

If the sequence $a_{n}$ in 1.1 above satisfies (i) $a_{n} n \rightarrow 1$ (ii) $a_{n} n \rightarrow 0$ and (iii) $a_{n} n \rightarrow \infty$ we call $X$ sparse, subcritical and supercritical respectively.

In this article we also assume that the sequence $\left(a_{n}\right)$ converges to 0 as $n$ approaches $\infty$.

For any finite or countable set $\mathcal{L}$, we denote by $\mathcal{L}(\Gamma)$ the space of probability vectors by $\tilde{\mathcal{L}}(\Gamma)$ space of finite positive measures on $\Gamma$, by $\tilde{\mathcal{L}}_{n}(\Gamma)$ we denote the subspace of symmetric measures in $\tilde{\mathcal{L}}(\Gamma)$. Вy $\mathcal{P}(\Gamma)$ the space of all real-valued bounded measurable functions on $\Gamma$, by $\mathcal{P}_{*}(\Gamma)$ the space of continuous linear functionals on $\mathcal{P}(\Gamma)$ and by $\mathcal{P}_{+}(\Gamma)$ the collection of all positive linear functional on $\mathcal{P}(\Gamma)$.

For every typed random graph $Y$, we define the empirical type distribution $L_{Y}^{1} \in \mathcal{L}(\Gamma)$ by:

$L_{Y}^{1}(a)=\frac{1}{n} \sum_{v \in[n]} \delta_{Y(v)}(a)$, for $a \in \Gamma$

and the empirical link distribution $L_{Y}^{2} \in \tilde{\mathcal{L}}_{*}(\Gamma \times \Gamma)$ is defined by:

$L_{Y}^{2}(a, b)=\frac{1}{a_{n} n^{2}} \sum_{(u, v) \in E}\left[\delta_{(Y(v), Y(u))}+\delta_{(Y(u), Y(v))}\right](a, b)$, for $a, b \in \Gamma$.

Note that $a_{n} n^{2}$ is the maximum possible number of edges in the graph and we have that:

$a_{n} n^{2} L_{X}^{2}(a, b)=\left\{\begin{array}{c}\#\{\text { number of edges between } \\ \text { vertices of colours } a \text { and } b\} \\ 2 \times \#\{\text { number of edges between } \\ \text { vertices of colours } a\}\end{array}\right.$ if $a=b$

The rest of the article is structured as follows: Section 2 contain the main results of the article (Theorem 2.1, Theorem 2.3, Corollary 2.2). Section 3 contain proofs of the main results of the article.

\section{Statement of Main Results}

We assume through out the remaining part of this article that the typed random graph process is nearcritical or sparse. We shall write $\langle f, \sigma\rangle:=\sum_{y \in \Gamma} \sigma(y) f(y)$ and define the spectral potential $\rho_{\lambda}(g, \mu)$ of the near-critical typed random graph process $Y$ by:

$$
\rho_{\lambda}(g, \mu)=-\left\langle\left(1-e^{g}\right), \lambda_{\mu \otimes \mu}\right\rangle / 2,
$$

where $\lambda_{\mu \otimes \mu}(a, b)=\lambda(a, b) \mu(a) \mu(b), \forall a, b \in \Gamma \times \Gamma$

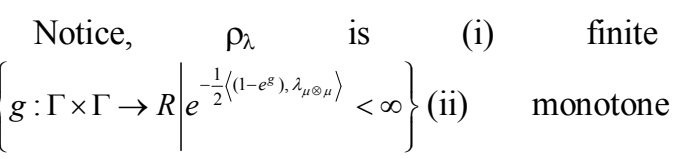

additively homogeneous and convex in $g$. For $v \in \mathcal{P}(\Gamma \times \Gamma)$ we define the Kullback action by a nonlinear functional:

$$
\mathcal{H}_{\lambda}(\pi \| \mu):=\left(\left\langle\pi, \log \frac{\pi}{\lambda_{\mu \otimes \mu}}\right\rangle+\left\|\lambda_{\mu \otimes \mu}\right\|-\|\pi\|\right) / 2
$$

and note that $\mathcal{H}_{\lambda}$ above is nonlinear functional. 
Let $P_{\mu}(y)=\mathbb{P}\left\{Y=y \mid L_{y}^{1}=\mu\right\}$ be the probability law of the near-critical typed random graph process $y$ on $[n]$ conditioned on the empirical type measure $\mu$. In Theorem 2.1 below we present our main result, the LLDP for the typed random graph process.

\section{Theorem 2.1.}

Let $y=(y(v): v \in[n])$ be a typed random graph process with type law $\mu$ and link probabilities that satisfies $a_{n}^{-1} p_{n}(a, b) \rightarrow \lambda(a, b)$, for $a, b \in \Gamma$ and $n a_{n} \rightarrow 1$. Then:

- for any $\omega \in \tilde{\mathcal{L}}(\Gamma \times \Gamma)$ and a number $\varepsilon>0$, there exists a weak neighborhood $\mathrm{B}_{\omega}$ such that:

$$
P_{\mu}\left\{y \in \mathcal{G}([n], \Gamma) \mid L_{y}^{2} \in B_{\omega}\right\} \leq e^{-n \mathcal{H}_{\lambda}(\pi \mid \mu)-n \varepsilon}
$$

- for any $\omega \in \tilde{\mathcal{L}}_{*}(\Gamma \times \Gamma)$, a number $\varepsilon>0$ and a fine neighborhood $B_{\omega}$ we have the asymptotic estimate:

$$
P_{\mu}\left\{y \in \mathcal{G}([n], \Gamma) \mid L_{y}^{2} \in B_{\omega}\right\} \geq e^{-n \mathcal{H}_{\lambda}(\pi \mid \mu)-n \varepsilon} .
$$

Next we state a corollary of Theorem 2.1, the McMillianBreiman Theorem for the typed random graph process. We define an entropy by:

$$
h^{\lambda}(\pi):=\left(\|\pi\|-\left\|\lambda_{\mu \otimes \mu}\right\|-\left\langle\pi, \log \frac{\pi}{\lambda_{\mu \otimes \mu}}\right\rangle\right) / 2 .
$$

\section{Corollary 2.2.}

Let $\mathcal{G}(\mid n], \Gamma)$ be the space of all typed random graph process with type law $\mu$ and link probabilities that satisfies $a_{n}^{-1} p_{n}(a, b) \rightarrow \lambda(a, b)$, for $a, b \in \Gamma$ and $n a_{n} \rightarrow 1$.

- For any empirical link measure $\rho$ on $\Gamma \times \Gamma$ and $\varepsilon>0$, there exists a neighborhoo $d B_{\rho}$ such that:

$$
\#\left\{y \in \mathcal{G}([n], \Gamma) \mid L_{y}^{2} \in B_{\rho}\right\} \geq e^{n\left(h^{\lambda}(\rho)+\varepsilon\right)} .
$$

- for any neighborhood $B_{\rho}$ and $\varepsilon>0$, we have:

$$
\#\left\{y \in \mathcal{G}([n], \Gamma) \mid L_{y}^{2} \in B_{\rho}\right\} \leq e^{n\left(h^{\lambda}(\rho)-\varepsilon\right)},
$$

where, \#\{A\} means the cardinality of $A$ :

\section{Remark 1}

For $\rho=\lambda_{\mu \otimes \mu}$, equation 2.3 above reduces to $h^{\lambda}(\pi):=-\left\langle\lambda_{\mu \otimes \mu}, \log \frac{\lambda_{\mu \otimes_{\mu}}}{\left\|\lambda_{\mu{ }_{\mu}}\right\|}\right\rangle$ and therefore, we have:

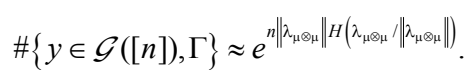

We state in Theorem 2.3 the full LDP for the typed random graph process below:

\section{Theorem 2.3.}

Let $y=(y(v): v \in[n])$ be a typed random graph process with type law $\mu$ and link probabilities that satisfies $a_{n}^{-1} p_{n}(a, b) \rightarrow \lambda(b, a)$, for $b, a \in \Gamma$ and $n a_{n} \rightarrow 1$.

- Let $F$ be open subset of $\tilde{\mathcal{L}}_{*}(\Gamma \times \Gamma)$. Then:

$$
\lim _{n \rightarrow \infty} \frac{1}{n} \log P_{\mu}\left\{y \in \mathcal{G}([n], \Gamma) \mid L_{y}^{2} \in F\right\} \geq-\inf _{\pi \in F} \mathcal{H}_{\lambda}(\pi \| \mu) .
$$

- Let $G$ be closed subset of $\tilde{\mathcal{L}_{*}}(\Gamma \times \Gamma)$. Then:

$$
\lim _{n \rightarrow \infty} \frac{1}{n} \log P_{\mu}\left\{y \in \mathcal{G}([n], \Gamma) \mid L_{y}^{2} \in G\right\} \leq-\inf _{\pi \in \Gamma} \mathcal{H}_{\lambda}(\pi \| \mu) .
$$

\section{Proof of Main Results}

\section{Properties of the Rate Function}

In this subsection Lemma 3.1, which summaries the properties of 2.2 above. This will help us circumvent the topological problems faced by Doku-Amponsah (2017b), Dembo et al. (2005). Denote by $C$ is the space of continuous functions $g: \Gamma \times \Gamma \rightarrow \mathbb{R}$.

\section{Lemma 3.1.}

The following holds for the Kullback action or divergence function $\mathcal{H}_{\lambda}(\pi \| \mu)$ :

- $\mathcal{H}_{\lambda}(\pi \| \mu)=\frac{1}{2} \sup _{g \in C}\left\{\langle g, \pi\rangle-\rho_{\lambda}(g, \mu)\right\}$

- The function $\mathcal{H}_{\lambda}(\pi \| \mu)$ is lower semi-continuous on the space $\mathcal{P}_{*}(\Gamma \times \Gamma)$

- For any real $c$, the set $\left\{v \in \mathcal{P}_{*}(\Gamma \times \Gamma): \mathcal{H}_{\lambda}(\pi \| \mu) \leq c\right\}$ is weakly compact

Please we refer to (Bakhtin, 2015) for similar result and proof for the empirical measures on measurable spaces.

The proof presented below follows similar ideas as the proof of [(Bakhtin, 2015), Lemma 2.2] for the empirical measures on measurable spaces.

\section{Proof}

(i) Let $g \in C$ be such that $\langle g, \pi\rangle$ estimates the functional $\langle\phi, \pi\rangle$ and $\rho_{\lambda}(g, \mu)$ estimates $\rho_{\lambda}(\phi, \mu)$ where $\phi \in \mathcal{P}(\Gamma \times \Gamma)$. Suppose $\pi$ is absolutely continuous with respect to $\lambda_{\mu \otimes \mu}$. 
Define the function $g$ by $g:=\log \frac{\pi}{\lambda_{\mu \otimes \mu}}$. For $t>0$, we define the estimate of $g, g_{t} \in \mathcal{P}(\Gamma \times \Gamma)$ as follows:

$g_{t}(a, b):=\left\{\begin{array}{cc}g(a, b), & \text { if }-t<g(a, b)<t, \\ e^{t}, & \text { if } g(a, b)>t \\ e^{-t}, & \text { if } g(a, b)<-t\end{array}\right.$

for all $(a, c) \in \Gamma \times \Gamma^{*}$. Now for $\mathrm{t} \rightarrow \infty$ we have that:

$\left\langle\left(1-e^{g t}\right), \lambda_{\mu \otimes \mu}\right\rangle=\int\left(1-e^{g(a, b)}\right) 1 l_{\{-t<g(a, b)<t\}} \lambda_{\mu \otimes \mu}(d a, d b)$

$+\int t 1 l_{\{g(a, b)>t\}} \lambda_{\mu \otimes \mu}(d a, d b)$

$+\int-t 1 l_{\{-t>g\}} \lambda_{\mu \otimes \mu}(d a, d b) \rightarrow\left\langle\left(1-e^{g}\right), \lambda_{\mu \otimes \mu}\right\rangle=\left\|\lambda_{\mu \otimes \mu}\right\|-\|\pi\|$

$\left\langle g_{t}, \pi\right\rangle=\int g 1 l_{\{-t<g<t\}} \pi(d a, d b)+\int t 1 l_{\{g>t\}} \pi(d a, d b)$

$+\int-t 1 l_{\{-t>g\}} \pi(d a, d b)$

$\rightarrow\langle g, \pi\rangle=\left\langle\pi, \log \frac{\pi}{\lambda_{\mu \otimes \mu}}\right\rangle$.

Therefore we

$\lim _{t \rightarrow \infty} \frac{1}{2}\left(\left\langle g_{t}, \pi\right\rangle-\left\langle\left(1-e^{g t}\right), \lambda_{\mu \otimes \mu}\right\rangle\right) \rightarrow \mathcal{H}_{\lambda}(\pi \| \mu)$ which

have proves

Lemma 3.1 (i). Suppose $\pi$ is not absolutely continuous with respect to $\lambda_{\mu \otimes \mu}$. i.e., there exists an $\varepsilon>0$ such that for any $1>\eta>0$ there exists $B_{\eta} \subset \Gamma \times \Gamma^{*}$ with $\lambda_{\mu \otimes \mu}\left(B_{\eta}\right) \leq \eta /(1-\eta)$ and at the same time we have $\pi\left(B_{\eta}\right)>\varepsilon$. For this $\eta$ define the function:

$g_{\delta}(a, b):=\left\{\begin{array}{cc}-\log \eta & \text { if }(a, b) \in B_{\eta}, \\ 0, & \text { if }(a, b) \notin B_{\eta} .\end{array}\right.$

Then

we

have $\lim _{\eta \downarrow 0} \frac{1}{2}\left(\left\langle g_{\eta}, \pi\right\rangle-\left\langle\left(1-e^{g \eta}\right), \lambda_{\mu \otimes \mu}\right\rangle\right) \geq \frac{\varepsilon}{2} \log \eta-\frac{1}{2}\left\langle\left(1-e^{g \eta}\right), \lambda_{\mu \otimes \mu}\right\rangle \geq-\frac{\varepsilon}{2} \log \eta+\frac{1}{2}$. Taking limit as $\eta \downarrow 0$ we have $\mathcal{H}_{\lambda}(\pi \| \mu)=+\infty$, which ends the proof of Lemma 3.1 (i). (ii) \& (iii). Observe from the properties of the relative entropy, see Dembo et al. (1998) and Lemma 3.1(i) that $\frac{1}{2} \sup _{g \in C}\left\{\langle g, \pi\rangle-\rho_{\lambda}(g, \mu)\right\}$ reduces to equation 2.2 above. Now the relative entropy is lower semi-continuous and by Doku-Amponsah (2012) all its level sets are compact. Hence it holds $\mathcal{H}_{\lambda}(\pi|| \mu)$ is lower semi-continuous and all its level sets are weakly compact in the weak topology which ends the proof of the Lemma.

Note that Lemma 3.1 (i) above implies the so-called variational principle. See, example Komkov (1986).

\section{Proof of Theorem 2.1.}

By Lemma 3.1, for any $\varepsilon>0$ there exists a function $g \in \mathcal{P}(\Gamma \times \Gamma)$ such that:

$$
\mathcal{H}_{\lambda}(\pi \| \mu)-\frac{\varepsilon}{2}<\langle g, \pi\rangle-\rho_{\lambda}(g, \mu)
$$

We define the probability distribution $\tilde{P}_{n}$ by:

$$
\begin{aligned}
& \tilde{P}_{\mu}(Y)=\prod_{(u, v) \in E} \tilde{n}_{n}(Y(u), Y(v)) \prod_{(u, v) \in E} 1-\tilde{p}_{n}(Y(u), Y(v)) \\
& =\prod_{(u, v) \in E} \frac{\tilde{p}_{n}(Y(u), Y(v))}{n-n \tilde{p}_{n}(Y(u), Y(v))} \prod_{(u, v) \in E}\left(n-n \tilde{p}_{n}(Y(u), Y(v))\right) \\
& =\prod_{(u, v) \in E} e^{g(Y(u,), Y(v))} \frac{p_{n}(Y(u), Y(v))}{n-n p_{n}(Y(u), Y(v))} \prod_{(u, v) \in E} e^{\frac{1}{i_{n}}\left(\tilde{n}_{n}(Y(u), Y(v))\right.}\left(n-n p_{n}(Y(u), Y(v))\right)
\end{aligned}
$$

Using 3.3 above, we have:

$$
\begin{aligned}
& \frac{d P_{\mu}(Y)}{d \tilde{P}_{\mu}(Y)}=\prod_{(u, v) \in E} e^{-g(Y(u), Y(v))} \\
& \prod_{(u, v) \in \varepsilon} e^{-\frac{1}{n} \tilde{h}_{n}(Y(u), Y(v))}=e^{-n\left\langle\frac{1}{2} L^{2}, \tilde{g}\right\rangle-n\left\langle\frac{1}{2} L^{1} \otimes L^{1}, \tilde{h}_{n}\right\rangle+\left\langle\frac{1}{2} L_{\Delta}^{1}, L^{1}, \tilde{h}_{n}\right\rangle},
\end{aligned}
$$

While:

$$
L_{\Delta}^{1}=\delta_{(Y(u), Y(u))} .
$$

Now we define a neighbourhood of the functional $\pi$ as follows:

$$
B_{\pi}=\left\{\varpi \in \mathcal{P}(\Gamma \times \Gamma):\langle g, \varpi\rangle>\langle g, \pi\rangle-\frac{\varepsilon}{2}\right\} .
$$

Therefore, under the condition $L_{y}^{1} \in B_{v}$ we have that:

$$
\frac{d P_{\mu}(x)}{d \tilde{P}_{\mu}(x)}<e^{\frac{1}{2}\left(\rho_{\lambda}(g, \mu)-\langle g, \pi)\right)+\frac{\varepsilon}{2}}<e^{-n H_{\lambda}(\pi \mid \mu)+n \varepsilon} .
$$

Hence, we have:

$$
\begin{aligned}
P_{\mu}\left\{y \in \mathcal{G}([n], \Gamma) \mid L_{y}^{2} \in B_{\pi}\right\} & \leq \int_{\mathcal{G}([n], \Gamma)} 1 l_{\left\{L_{y}^{2} \in B_{v}\right\}} d \tilde{P}_{\mu}(x) \\
& \leq \int_{\mathcal{G}([n], \Gamma)} 1 l_{\left\{L_{y}^{2} \in B_{\pi}\right\}} e^{-n \mathcal{H}_{\lambda}(\pi \| \mu)-n \varepsilon} d \tilde{P}_{\mu}(x) \\
& \leq e^{-n \mathcal{H}_{\lambda}(\pi \| \mu)-n \varepsilon}
\end{aligned}
$$

Note that $\mathcal{H}_{\lambda}(\pi \| \mu)=\infty$ implies Theorem 2.1(ii) and so it suffice to prove that for a probability measure of the form $\pi=e^{g} \lambda_{\mu \otimes \mu}$, where the Kullback action $\mathcal{H}_{\lambda}(\pi \| \mu)=$ $\langle g, \pi\rangle+\left\langle\left(1, e^{g}\right), \lambda_{\mu \otimes \mu}\right\rangle$ is finite. Fix any number $\varepsilon>0$ and any neighbourhood $\mathrm{B}_{\pi} \subset \mathcal{L}(\Gamma \times \Gamma)$. We define the sequence of sets:

$$
\tilde{\mathcal{G}}([n], \Gamma):=\left\{y \in \mathcal{G}([n], \Gamma): L_{y}^{2} \in B_{v}\left|\left\langle g, L_{y}^{2}\right\rangle-\langle g, \pi\rangle\right| \leq \frac{\varepsilon}{2}\right\} .
$$

Observe that, for all $x \in \mathcal{G}([n], \Gamma)$ we have: 


$$
\frac{d P_{\mu}(x)}{d \tilde{P}_{\mu}(x)}=e^{-n\left(\frac{1}{2} L^{2}, \tilde{g}\right)-n\left(\frac{1}{2} L^{1} \otimes L^{1}, \tilde{h}_{n}\right\rangle+\left\langle\left(\frac{1}{2} L_{\perp}^{1}, \tilde{h}_{n}\right\rangle\right.}>e^{-n\left(\frac{1}{2} \pi, \log \frac{\pi}{\lambda_{\mu \otimes \mu}}\right)-n \frac{1}{2}\left\langle\lambda_{\mu \otimes \mu},\left(1-\frac{\pi}{\lambda_{\mu \otimes \mu}}\right)\right\rangle}
$$

This gives:

$$
\begin{aligned}
P_{\mu}(\tilde{\mathcal{G}}([n], \Gamma)) & =\int_{\tilde{\mathcal{G}}([n], \Gamma)} d P_{\mu}(x) \\
& \geq \int_{\tilde{\mathcal{G}}([n], \Gamma)} e^{\left.-\left\langle\frac{1}{2} \pi, \log \frac{\pi}{\lambda_{\mu \otimes \mu}}\right)-n \frac{1}{2} / \lambda_{\mu \otimes \mu},\left(1-\frac{\pi}{\lambda_{\mu \otimes \mu}}\right)\right)+\frac{\varepsilon}{2}} d \tilde{P}_{\mu}(x) \\
& =e^{-n \mathcal{H}_{\lambda}(\pi \| \mu)+n \varepsilon} \tilde{P}_{\mu}(\tilde{\mathcal{G}}([n], \Gamma)) .
\end{aligned}
$$

Using the law of large numbers we have $\lim _{n \rightarrow \infty} \tilde{P}_{\mu}(\tilde{\mathcal{G}}([n], \Gamma))=1$ which completes the proof.

\section{Proof of Corollary 2.2 and Theorem 2.3.}

\section{Proof of Corollary 2.2.}

The proof of Corollary 2.2 follows from the definition of the Kullback action and Theorem 2.1 if we set $\pi=\rho$ and $\lambda_{\mu \otimes \mu}(a, b)=\left\|\lambda_{\mu \otimes \mu}\right\|$, for all $(a, b) \in \Gamma \times \Gamma$.

The proof of Theorem 2.3 below, follows from Theorem 2.1 above using similar arguments as in [(Bakhin, 2015), p. 544].

\section{Proof of Theorem 2.3.}

\section{Proof}

Note that the empirical link measure is a finite measure and so belongs to some ball in $\mathcal{B} *(\Gamma \times \Gamma)$. Hence, without loss of generality we may assume that the set $\Gamma$ in Theorem 2.3(ii) is relatively compact. See Lemma 3.1 (iii). Choose any $\varepsilon>0$. Then for every functional $\pi \in \Gamma$ we can find a weak neighbourhood such that the estimate of Theorem 2.1(i) holds. We choose from all these neighbourhood a finite cover of $\mathcal{G}([n], \Gamma)$ and sum up over the estimate in Theorem 2.1(i) to obtain:

$$
\lim _{n \rightarrow \infty} \frac{1}{n} \log P_{n}\left\{y \in \mathcal{G}([n], \Gamma) \mid L_{y}^{2} \in G\right\} \leq-\inf _{\pi \in \Gamma} \mathcal{H}_{\lambda}(\pi \| \mu)+\varepsilon .
$$

As $\varepsilon$ was arbitrarily chosen and the lower bound in Theorem 2.1(ii) implies the lower bound in Theorem 2.3(i) we have the desired results which ends the proof of the Theorem.

\section{Conclusion}

In this article, we have found a Local Large Deviation Principle (LLDP) for the typed random graph processes using techniques rooted in spectral potential theory. From this LLDP we have proved a weak variant of the famous McMillian Theorem and a full conditional LDP for the typed random graph model. These results like the ones in Bakhtin (2015), Doku-Amponsah (2017c) differ from those in O'Connell (1998), Biggins and Penman (2009), Doku-Amponsah and Mörters, (2010), Bordenave and Caputo (2015), Doku-Amponsah (2012; 2017a) by the limited or no topology requirement for the space of finite graphs. This result has thrown more light on the basic information theorems developed for coloured random graphs in (Doku-Amponsah, 2012).

\section{Acknowledgement}

This paper was prepared at the Carnigie BangaAfrica, June 27-July 2017 write-shop organized at Koforidua.

\section{Ethics}

The author declares that there is no conflict of interest.

\section{References}

Bakhtin, I.V., 2015. Spectral potential, kullback action and large deviations of empirical measureson measureable spaces. Theory Probability Applic., 50: 535-544.

Biggins, J.D. and D.B. Penman, 2009. Large deviations in randomly coloured random graphs. Electron. Commun. Probab., 29: 290-301.

Bordenave, C. and P. Caputo, 2015. Large deviations of empirical neighborhood distribution in sparse random graphs. Probability Theory Related Fields, 163: 149-222.

Cannings, C. and D.B. Penman, 2003. Models of Random Graphs and their Applications Handbook of Statistics 21. In: Stochastic Processes: Modeling and Simulation. Shanbhag, D.N. and C.R. Rao, (Eds.), Elsevier, pp: 51-91.

Doku-Amponsah, K., 2017a. Lossy asymptotic equipartition property for networked data structures. J. Math. Stat., 13: 152-158.

Doku-Amponsah, K., 2017b. Lossy asymptotic equipartition property for hierarchical data structures. Far East J. Math. Sci., 101: 1013-1024.

Doku-Amponsah, K., 2017c Local large deviations, McMillian theorem for multitype Galton-Watson processes. Far East J. Math. Sci. 102: 2307-2319.

Doku-Amponsah, K., 2012. Asymptotic equipartition properties for hierarchical and networked structures. ESAIM: Probability Stat., 16: 114-138.

Doku-Amponsah, K. and P. Mörters, 2010. Large deviation principles for empirical measures of colored random graphs. Ann. Applied Probability, 20: 1989-2021.

Dembo, A., P. Mörters and S. Sheffield, 2005. Large deviations of Markov chains indexed by random trees. Ann. Inst. Henri Poincare: Probab.et Stat., 41: 971-996. DOI: 10.1016/j.anihpb.2004.09.005 
Dembo, A. and O. Zeitouni, 1998. Large Deviations Techniques and Applications. 2nd Edn., Springer Science and Business Media, Berlin, ISBN-10: 3642033113, pp: 396.

Komkov, V., 1986. Variational principles of continuum mechanics with engineering applications. Vol. 1. Critical points theory. Mathematics and its Applications, 24. D. Reidel Publishing Co., Dordrecht.
O'Connell, N., 1998. Some large deviation results for sparse random graphs. Probab. Theory Relat. Fields, 110: 277- 285.

Penman, D.B., 1998. Random graphs with correlation structure. PhD Thesis, Sheffield. 